\title{
MicroRNA as a novel player in atrial fibrillation
}

\author{
Siyi Fu ${ }^{1,2+}$, Leqi Huang ${ }^{1,2+}$, Yalong Wang ${ }^{1,2+}$, Xing $\mathrm{Li}^{1,2}$, Jie $\mathrm{Li}^{1,2}$ and Junjie Xiao ${ }^{1,2,3 *}$ \\ ${ }^{1}$ Regeneration Lab and Experimental Center of Life Sciences, School of Life Science, Shanghai University, Shanghai, China \\ 2 Shanghai Key Laboratory of Bio-Energy Crops, School of Life Sciences, Shanghai University, Shanghai, China \\ ${ }^{3}$ Innovative Drug Research Center of Shanghai University, Shanghai, China \\ *Correspondence: junjiexiao@live.cn
}

Edited by:

Yi-Qing Yang, Medical College of Shanghai Jiao Tong University, China

Reviewed by:

Xinli Li, Nanjing Medical School, China

${ }^{\dagger}$ These authors have contributed equally to this work.

Keywords: microRNA, atrial fibrillation, electrial remodeling, structural remodeling, therapy

Atrial fibrillation (AF), with an extremely highly age-dependent prevalence, is a most frequent type of sustained arrhythmia in the clinic both in developing and developed countries (Dobrev and Nattel, 2011). AF patients have a lot of symptoms including palpitations, dizziness, breathlessness, and chest pain. AF will lead to an increased risk of stroke and aggravate congestive heart failure (Shi et al., 2013). Compared with familial AF, the non-familial AF occupies the majority of AF. In China, at least 10 million people are affected by it. Therefore, AF is associated with a remarkable morbidity and mortality, leading to a large socio-economic burden. Unfortunately, the underling mechanisms of AF are still exclusive (Wang et al., 2011; Liu et al., 2012a,b).

Several hypotheses have been proposed for AF. Focal activity, single-circuit reentry, and multiple-circuit reentry have been generally considered as three classic models for AF. In addition, different substrates which facilitate the formation of AF have also been revealed including electrical remodeling, structure remodeling, and intracellular $\mathrm{Ca}^{2+}$ handling remodeling. Moreover, various genetic mutations such as chromosomal loci (10q22-q24 and 6q14-16) mutations, ion channel $\left(\mathrm{Ka}^{+}\right.$ and $\mathrm{Na}^{+}$) mutations, connexin (GJA5 and GJA1) mutations in the familial $\mathrm{AF}$ and more recently several common variants on $4 \mathrm{q} 25,16 \mathrm{q} 22$, and $1 \mathrm{q} 21$ in the nonfamilial AF have been identified (Xiao et al., 2011a,b; Liu et al., 2012a,b). Despite this, the definitive elucidation of AF still remains relatively limited and unclear (Zhang et al., 2011).
MicroRNAs (miRNAs, miRs) are a novel class of endogenous non-coding RNAs of around 22 nucleotides in length, which are widely accepted to possess a key role in the gene expression regulatory network at the post-transcriptional level (Wang et al., 2011; Fu et al., 2013). So far, at least one thousand miRNAs have been identified and they have been reported to participate in many fundamental biological processes including cell proliferation, growth, differentiation, apoptosis, and tissue remodeling. Dysregulated miRNAs have been shown to be related to the genesis of many cardiovascular diseases, including arrhythmia, hypertrophy, and heart failure. Moreover, miRNAs have also been identified to be necessary for the differentiation of human-derived cardiomyocyte progenitor cells (Xiao et al., 2012). Furthermore, circulating miRNAs can also be served as promising biomarkers for acute myocardial infarction and heart failure etc. (Dimmeler and Zeiher, 2010; Li et al., 2013). Thus, it is not surprising that miRNAs gain a critical position in the physiological and pathological processes of the cardiovascular system (Wang et al., 2011; Xiao et al., 2011a,b; Liu et al., 2012a,b).

Several studies have presented interesting connections between miRNAs and AF. Of note, a group of miRNAs has been identified to regulate target genes encoding cardiac ion channels/transporters/ $/ \mathrm{Ca}^{2+}$ handling proteins, which may participate in the genesis of AF (Wang et al., 2011; Dobrev, 2012). Interestingly, many available data have demonstratedthatmiR1, miR-21, miR-26, miR-29, miR-30,
miR-133, miR-208, miR-328, miR-499, and miR-590 might take part in the genesis of AF (Dawson et al., 2013; Shi et al., 2013). Most of these miRNAs promote the electrical or structural remodeling in the atrium. MiR-26 family contributes to $\mathrm{AF}$ via repressing the expression of $\mathrm{KCNJ} 2 / \mathrm{Kir} 2.1 / \mathrm{I}_{\mathrm{K} 1}$ while miRNA-1 via regulating $\mathrm{I}_{\mathrm{K} 1}$ expression and $\mathrm{Ca}^{2+}$ handling proteins. In addition, miR-499 regulates $\mathrm{KCNN} 3 / \mathrm{SK} 3$ while miR-328 targets CACNA1C and CACNB, contributing to adverse electrical remodeling ( $\mathrm{Lu}$ et al., 2010). Moreover, miR-133 and miR-590 have been found to target transforming growth factor- $\beta 1$ (TGF- $\beta 1$ ) and TGF$\beta$ receptor type II (TGF- $\beta$ RII), leading to structure remodeling in AF (Shan et al., 2009). Interestingly, circulating levels of miR-328 and miR-150 have been reported to be down-regulated in $\mathrm{AF}$ patients though the functional role is still unclear (Liu et al., 2012a,b; McManus et al., 2014).

With more systematic and insightful studies exploring the miRNAs basis for AF, novel therapeutics will be developed and ultimately lead to advanced treatment (Zhao et al., 2013).

\section{ACKNOWLEDGMENTS}

This work was supported by the grants from National Natural Science Foundation of China(81200169 to Junjie Xiao), Innovation Program of Shanghai Municipal Education Commission (13YZ014 to Junjie Xiao), Foundation for University Young Teachers by Shanghai Municipal Education Commission (year 2012, to Junjie Xiao), Innovation 
Foundation of Shanghai University (sdcx2012038, to Junjie Xiao).

\section{REFERENCES}

Dawson, K., Wakili, R., Ördög, B., Clauss, S., Chen, Y., Iwasaki, Y., et al. (2013). MicroRNA29: a mechanistic contributor and potential biomarker in atrial fibrillation. Circulation 127, 1466-1475, 1475e1-1475e28. doi: 10.1161/ CIRCULATIONAHA.112.001207

Dimmeler, S., and Zeiher, A. (2010). Circulating microRNAs: novel biomarkers for cardiovascular diseases? Eur. Heart J. 31, 2705-2707. doi: 10.1093/ eurheartj/ehq221

Dobrev, D. (2012). Is altered atrial microRNA-ome a critical contributor to the pathophysiology of atrial fibrillation? Basic Res. Cardiol. 107, 284. doi: 10.1007/s00395-012-0284-2

Dobrev, D., and Nattel, S. (2011). New insights into the molecular basis of atrial fibrillation:mechanistic and therapeutic implications. Cardiovasc. Res. 89, 689-691. doi: 10.1093/cvr/ cvr021

Fu, S., Zhuo, R., Yao, M., Zhang, J., Zhou, H., and Xiao, J. (2013). MicroRNA basis of physiological hypertrophy. Front. Genet. 4:253. doi: 10.3389/ fgene.2013.00253

Li, J., Xu, J., Cheng, Y., Wang, F.,Song, Y., and Xiao, J. (2013). Circulating microRNAs as mirrors of acute coronary syndromes: MiRacle or quagMire? J. Cell. Mol. Med. 17, 1363-1370. doi: 10.1111/ jcmm.12148

Liu, X., Wang, F., Knight, A., Zhao, J., and Xiao, J. (2012a). Common variants for atrial fibrillation: results from genome-wide association studies. Hum. Genet. 131, 33-39. doi: 10.1007/s00439-0111052-3
Liu, Z., Zhou, C., Liu, Y., Wang, S., Ye, P., Miao, X., et al. (2012b). The expression levels of plasma micoRNAs in atrial fibrillation patients. PLOS ONE 7:e44906. doi: 10.1371/journal.pone.0044906

Lu, Y., Zhang, Y., Wang, N., Pan, Z., Gao, X., Zhang, F., et al. (2010). MicroRNA-328 contributes to adverse electrical remodeling in atrial fibrillation. Circulation 122, 2378-2387. doi: 10.1161/ CIRCULATIONAHA.110.958967

McManus, D. D., Lin, H., Tanriverdi, K., Quercio, M., Yin, X., Larson, M. G., et al. (2014). Relations between circulating microRNAs and atrial fibrillation: data from the framingham offspring study. Heart Rhythm. 11, 663-669. doi: 10.1016/j.hrthm. 2014.01.018

Shan, H., Zhang, Y., Lu, Y., Zhang, Y., Pan, Z., Cai, B., et al. (2009). Downregulation of miR133 and miR-590 contributes to nicotine-induced atrial remodelling in canines. Cardiovasc. Res. 83, 465-472. doi: 10.1093/cvr/cvp130

Shi, K. H., Tao, H., Yang, J. J., Wu, J. X., Xu, S. S., and Zhan, H. Y. (2013). Role of microRNAs in atrial fibrillation: new insights and perspectives. Cell Signal. 25, 2079-2084. doi: 10.1016/j.cellsig. 2013.06.009

Wang, Z., Lu, Y., and Yang, B. (2011). MicroRNAs and atrial fibrillation: new fundamentals. Cardiovasc. Res. 89, 710-721. doi: 10.1093/cvr/cvq350

Xiao, J., Liang, D., and Chen, Y. (2011a). The genetics of atrial fibrillation: from the bench to the bedside. Annu. Rev. Genomics Hum. Genet. 12, 73-96. doi: 10.1146/annurev-genom-082410101515

Xiao, J., Liang, D., Zhang, Y., Liu, Y., Zhang, H., Liu, Y., et al. (2011b). MicroRNA expression signature in atrial fibrillation with mitral stenosis. Physiol. Genomics 43, 655-664. doi: 10.1152/ physiolgenomics.00139.2010
Xiao, J., Liang, D., Zhang, H., Liu, Y., Zhang, D., Liu, Y., et al. (2012). MicroRNA-204 is required for differentiation of human-derived cardiomyocyte progenitor cells. J. Mol. Cell. Cardiol. 53, 751-759. doi: 10.1016/j.yjmcc.2012.08.024

Zhang, Y., Dong, D., and Yang, B. (2011). Atrial remodeling in atrial fibrillation and association between microRNA network and atrial fibrillation. Sci. China Life Sci. 54, 1097-1102. doi: 10.1007/ s11427-011-4241-3

Zhao, Z., Liu, T., Wang, X., and Li, G. (2013). MicroRNAs as novel antiarrhythmic targets for atrialfibrillation. Int. J. Cardiol. 168, e135-e137. doi: 10.1016/j.ijcard.2013.08.014

Conflict of Interest Statement: The authors declare that the research was conducted in the absence of any commercial or financial relationships that could be construed as a potential conflict of interest.

Received: 20 February 2014; accepted: 05 April 2014; published online: 30 April 2014

Citation: Fu S, Huang L, Wang Y, Li X, Li J and Xiao J (2014) MicroRNA as a novel player in atrial fibrillation. Front. Genet. 5:97. doi: 10.3389/fgene.2014.00097

This article was submitted to Epigenomics and Epigenetics, a section of the journal Frontiers in Genetics.

Copyright (c) 2014 Fu, Huang, Wang, Li, Li and Xiao. This is an open-access article distributed under the terms of the Creative Commons Attribution License (CC BY). The use, distribution or reproduction in other forums is permitted, provided the original author(s) or licensor are credited and that the original publication in this journal is cited, in accordance with accepted academic practice. No use, distribution or reproduction is permitted which does not comply with these terms. 